\title{
Capturing the Signature of Topological Evolution from the Snapshots of Road Networks
}

\author{
Gi Ung Jang, ${ }^{1,2}$ Jin Chul Joo $\mathbb{D}^{3},^{3}$ and Jeryang Park $\mathbb{D}^{1}$ \\ ${ }^{1}$ Department of Civil Engineering, Hongik University, Seoul 04066, Republic of Korea \\ ${ }^{2}$ Water Supply and Sewage Project Office, Goyang City, Gyeongi-do 10460, Republic of Korea \\ ${ }^{3}$ Department of Civil and Environmental Engineering, Hanbat National University, Daejeon 34158, Republic of Korea
}

Correspondence should be addressed to Jeryang Park; jeryang@hongik.ac.kr

Received 30 October 2019; Accepted 19 March 2020; Published 25 April 2020

Academic Editor: Bernhard C. Geiger

Copyright ( $) 2020 \mathrm{Gi}$ Ung Jang et al. This is an open access article distributed under the Creative Commons Attribution License, which permits unrestricted use, distribution, and reproduction in any medium, provided the original work is properly cited.

Road networks serve as the backbone of cities, shaping urban structure as well as providing the critical function of transport for people, goods, and services. The design and management of resilient road infrastructure, therefore, is essential for building a sustainable city. Road networks grow and evolve over time, such that their topology shifts from an initially planned state to the one that emerges from self-organization and urban growth. In this work, we use a dual mapping approach to compare the topological features of road networks in 25 districts in Seoul, South Korea. By using average node degree as an indicator of the level of selforganization, we present that multiple topological variables including power-law exponent gradually shift as a network grows. By testing static error and attack tolerance of these networks, we also show that the gradual shift in topology also has an important implication in network resilience. We suggest a new method, inspired by Lorenz curve, for quantifying network vulnerability. This modified Lorenz curve enables calculating the relative impact of intensive attacks to random failures and shows that a more selforganized road network tends to become more vulnerable to selective attacks.

\section{Introduction}

A complex network is an abstract representation of a system, where system elements are treated as nodes, and node-to-node relationships are represented by links $[1,2]$. Despite being an abstract representation of reality, complex networks have been widely used to assess structure and function of many critical infrastructure systems including, but not limited to, roads [3-6], power grids [7-9], and water supply systems [10]. These examples of networks are composed of homogeneous or heterogeneous elements connected in a complex way, leading to emergent outcomes especially in macroscale that are difficult to predict from examining local properties. A seminal example for infrastructure systems is the Albert et al. [11] study of the topology of World Wide Web and Internet. In this work, they found nontrivial relationships between the topology of these networks and their tolerance to attack and error.

Here, we focus on relating the topology of urban road networks in South Korea to their relative level of self- organization and their tolerance to network disruptions. Previously, a considerable amount of effort has been made by the research community to understand the structure of urban road networks $[3,4,12-16]$. Several of these studies focus on correlating the topology of multiple road networks together to identify universality (e.g., scale-invariance) in their structure [3, 4, 13-15]. Nonetheless, few authors relate road network topology to the age of the infrastructure networks $[5,12,17,18]$. Several recent works categorized cities and their street networks into being self-organized (e.g., London) and top-down planned (e.g., Beijing) based on their current appearance and shape [19]. However, the road networks can also grow and evolve over time even after a highly centralized, topdown plan shaping the originally planned structure into a selforganized one. That is, a self-organized structure of a road network can be understood as a result from the spatiotemporal dynamics of city growth. Thus, the topological characteristics of newer, top-down road networks can vary considerably from older, self-organized systems [17]. 
Complex networks can be used to answer questions that relate road structure to their resilience, namely, "how vulnerable are roads to failures?" Urban road networks can be affected by natural hazards (e.g., floods, earthquakes,) that disrupt their critical services. Also, disruptions are often caused by man-made sources, such as the tight coupling between infrastructure systems within the same urban landscape (e.g., road networks overlay water supply and sewer networks in most cities [20-22]). For many cities in South Korea, there have been numerous reported cases of road subsidence caused by water leakages from aging water infrastructure that result in frequent traffic flow disruptions $[23,24]$. Taken together, the increasing frequency and intensity of natural disasters from climate change and manmade disruptions from aging infrastructure necessitate that sustainable and resilient road networks must also be robust to these future disruptions.

Vulnerability analysis of road networks can assist in determining critical nodes and links that influence overall system function. Not all disruptions are created equal, such that the loss of some infrastructure can have a greater impact on system function than others. From this perspective, many studies have investigated network topology to determine how node centralities (e.g., degree and betweenness) relate to the size and severity of network disturbances $[11,25]$. These studies developed different node or link removal strategies and measured their impact on the network function. This approach is also relevant for roads networks [19], as failures occurring in a structurally important location can result in greater adverse effects such as a longer duration of traffic congestions, a larger number of cars stuck in the traffic jam, and a wider cascade of the failure to other parts of the system. For instance, Zhan et al. [26] successfully showed how a functional failure of a road network by traffic congestion amplifies or is suppressed depending on the interaction of network topology and flow loading. Moreover, as the infrastructure grows and evolves, their centralities and topology also change resulting in different vulnerability characteristics over time. Therefore, it is important to analyze how the function of the network degrades relative to the where disruptions occur.

In this work, we assess the topology of 25 road networks in Seoul, Korea. In particular, we use a dual-mapping approach to explore how topology and network vulnerability to disruptions relate to road network age and geographic region. The paper is organized as follows: In Section 2, the road network data collection, dual mapping approach, calculation of network centrality metrics, and vulnerability analysis methods are described. Section 3 presents the results of our analysis, and associated discussions on how the topology of planned and self-organized networks from different geographic regions relate to one another are made in Section 4. Finally, in Section 5, we draw several conclusions.

\section{Data and Methods}

2.1. Urban Road Data. We selected 25 districts in Seoul with various levels of growth and self-organization for a comparative study (Figure 1). Through densification and

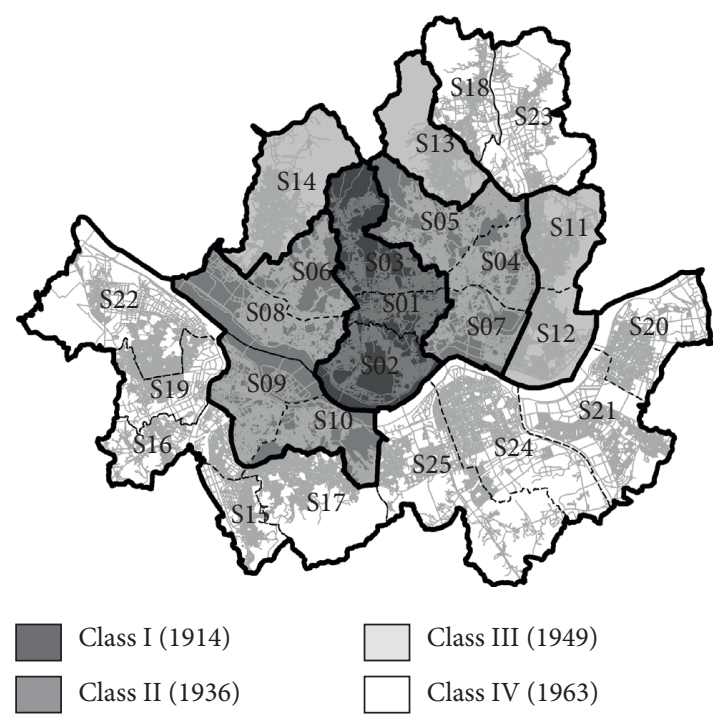

Figure 1: Twenty-five districts in Seoul classified by their development history.

exploration, the road networks in growing areas self-organize over time, causing a gradual shift in network topology [16]. In their initial design, urban road networks of Seoul are typically established by central and top-down planning with a grid structure to maximize the efficiency of flow. As the region experiences increasing population and densifying urban activities over time, road networks grow and evolve to suffice the increasing functional demand to transportation with continuous, local growth processes. But these processes are generally constrained by the existing geometry and landuse [17]. Various local factors such as geography, history, and economy, also play crucial roles in the evolution and growth of a city [27]. An initial condition for the formation of the infrastructure networks set by a central planning also produces distinct shapes in its earlier stage. However, considering the temporal scale (e.g., decades or centuries) for the growth and evolution of a city, obtaining long historical data of infrastructures $[5,6,17]$ is difficult due to a lack of digitized and georeferenced old maps in Korea. Instead, assuming that common properties of infrastructure networks in meso-to macro-scale can be found along its growth and evolution, we analyzed the snapshots of multiple urban sites which are currently in different stages of growth.

Table 1 summarizes the general characteristics-area of district $(A)$, number of nodes $\left(N_{\mathrm{p}}\right)$ and edges $\left(M_{\mathrm{p}}\right)$, and average node degree $\left.\left(<k_{\mathrm{p}}\right\rangle\right)$ of all districts. Note that the subscript $p$ denotes that metrics are measured in the primal mapping. The details on the network mapping (e.g., primal and dual) and their metrics are explained in Section 2.2. Based on the history of Seoul urban planning, by which the administrative boundary of Seoul was significantly expanded in each stage, we first attempted to categorize the districts by their age classes (Class I to IV). However, as our analysis unit is the district bounded by current administration boundaries (Figure 1), and since the regions that are expanded after every major urban plan do not exactly match with the current boundaries, our age classification is an 
TABle 1: Properties of urban road networks of 25 districts in Seoul, South Korea. $N_{\mathrm{p}}, M_{\mathrm{p}}$ and $<k_{\mathrm{p}}>$ are the number of nodes and edges, and average node degree, respectively, measured in the primal map.

\begin{tabular}{|c|c|c|c|c|c|c|}
\hline Age Class & Name & Sites & Area of district $\left(\mathrm{km}^{2}\right)^{\mathrm{a}}$ & $N_{\mathrm{P}}$ & $M_{\mathrm{P}}$ & $<k_{\mathrm{P}}>$ \\
\hline \multirow{3}{*}{ I } & S01 & Jung-gu & 10.0 & 3359 & 4470 & 2.66 \\
\hline & S02 & Yongsan-gu & 21.9 & 5534 & 6894 & 2.49 \\
\hline & S03 & Jongro-gu & 23.9 & 5340 & 6509 & 2.44 \\
\hline \multirow{7}{*}{ II } & S04 & Dongdaemun-gu & 14.2 & 6286 & 8095 & 2.58 \\
\hline & S05 & Seongbuk-gu & 24.6 & 10121 & 12786 & 2.53 \\
\hline & S06 & Seodaemun-gu & 17.6 & 4778 & 6120 & 2.56 \\
\hline & S07 & Seongdong-gu & 16.9 & 4576 & 5828 & 2.55 \\
\hline & S08 & Mapo-gu & 23.8 & 2860 & 4087 & 2.86 \\
\hline & S09 & Youngdeungpo-gu & 24.6 & 4916 & 6982 & 2.84 \\
\hline & S10 & Dongjak-gu & 16.4 & 4198 & 5289 & 2.52 \\
\hline \multirow{4}{*}{ III } & S11 & Jungrang-gu & 18.5 & 7723 & 9819 & 2.54 \\
\hline & S12 & Gwangjin-gu & 17.1 & 5859 & 7625 & 2.60 \\
\hline & S13 & Gangbuk-gu & 23.6 & 7455 & 9645 & 2.59 \\
\hline & S14 & Eunpyeong-gu & 29.7 & 4284 & 5772 & 2.69 \\
\hline \multirow{11}{*}{ IV } & S15 & Geumcheon-gu & 13.0 & 3416 & 4361 & 2.55 \\
\hline & S16 & Guro-gu & 20.1 & 5259 & 6710 & 2.55 \\
\hline & S17 & Gwanak-gu & 29.6 & 7175 & 9285 & 2.59 \\
\hline & S18 & Dobong-gu & 20.7 & 4137 & 5265 & 2.55 \\
\hline & S19 & Yangcheon-gu & 17.4 & 2810 & 4109 & 2.92 \\
\hline & S20 & Gangdong-gu & 24.5 & 3683 & 5015 & 2.72 \\
\hline & S21 & Songpa-gu & 33.9 & 4202 & 6166 & 2.93 \\
\hline & S22 & Gangseo-gu & 41.4 & 4974 & 6820 & 2.74 \\
\hline & S23 & Nowon-gu & 35.4 & 4006 & 5389 & 2.69 \\
\hline & $\mathrm{S} 24$ & Gangnam-gu & 39.5 & 3865 & 6098 & 3.16 \\
\hline & $\mathrm{S} 25$ & Seocho-gu & 47.0 & 4262 & 5989 & 2.81 \\
\hline
\end{tabular}

${ }^{\text {a }}$ source: stat.Seoul.go.kr.

approximation based on the proximity of the urban planning history.

The oldest age class (Class I) includes the districts from S01 to S03, where the areas are designated in the year 1914 by the Japanese Government-General of Korea during the Japanese colonial era. These areas have long been the center of the Korean peninsula over more than 1,000 years since the pre-modern periods. Although, the modern urban patterns of these areas have been affected by the historical development, the modern urban planning, which is the origin of the current shape of those areas, began after the 1936 Seoul Town Plan. Since then, expansion of the Seoul boundary and development of those newly included areas followed mainly centered in the Northern part of Seoul. By the 1936 Seoul Town Plan, the part of seven districts from S04 to S10 were included in Seoul and we categorized these districts as Class II. The third age class (Class III) from S11 to S14 were included in 1949. And the rest and the youngest districts from S15 to S25 were included in 1963, and we categorized those as Class IV.

GIS (Geographic information system) maps for road networks in the 25 districts as of 2014 are obtained from National Spatial Information Clearinghouse (NSIC, https:// www.nsic.go.kr). The original GIS data contained some errors such that the roads were separated at the intersection which were supposed to be connected. To fix the errors, we applied the tolerance level of 1 meter such that the end points of segments that are closer than the tolerance level are treated as connected.
2.2. Dual Mapping Approach. Two types of network representations are commonly used for roads: primal and dual approaches. While the primal approach defines the intersections as nodes and streets as edges [28], the dual approach defines the transportation units (e.g., an assemblage of road segments with the same identity) as nodes and intersections as edges [29]. It is more straightforward and intuitive to use the primal approach as it generates a network graph that is similar to the real road map and contains rich geographical features. However, the primal approach is limited in capturing the complexity and content-based structure of the system because street segments are not often constitutive units for transportation but rather the assemblages of such segments [5]. In contrast, the dual approach, or which is also called information space [5], is known to be better at capturing interesting topological properties of roads, such as small-world or scale-free properties $[4,5,30]$. Consequently, the dual approach also allows the comparison of road networks with other non-spatial, complex networks, such as social or biological networks. By treating roads that maintain "continuity" (defined below) as nodes and intersections as links, it is possible to have nodes with numerous edges (e.g., hubs) that could not otherwise exist in a primal mapping. For these reasons, the dual approach for mapping road networks is well accepted for capturing the complexity of urban systems [5].

In the dual mapping approach, it is first necessary to define what makes roads "continuous," i.e., the criteria for combining multiple street segments into a single "road unit" 
or node. There are multiple approaches for defining continuity of road units, including road segments with the same name are the same node [31] or connected road segments that share similar physical or geometric properties are the same node [29]. Here, we apply the latter approach with the chosen geometrical property as the linearity of road segments. Specifically, we define a continuous road unit as a combination of road segments connected by intersections whose maximum angular threshold between segments does not exceed $60^{\circ}$ [32]. Thus, at a given intersection (link), road segments with minor deviations in their relative angle are combined into a single road unit (node), where sharp turns or large changes in the direction are treated as separate road units. For this procedure, we used the open ArcGIS extension, Axwoman 6.3 [33]. The resulting network is then represented as a graph $G\left(N_{D}, M_{D}\right)$ which is composed of a set of nodes or vertices $\left(N_{D}\right)$ and another set of edges or links $\left(M_{D}\right)$ that describes the connectivity between the nodes. The obtained graph $G$ is expressed by an adjacency matrix $A$ whose element $a_{i j}$ is 1 if the node $i$ and $j$ are connected and 0 otherwise. We define the network as undirected since there are, if any, only a small portion of nodes are with one-way direction. Thus, the graph $G$ is a $N_{D} \times N_{D}$ symmetric matrix.

2.3. Network Metrics. Centrality metrics are used for identifying the most important nodes within a network by defining specific criteria. This information about the structural importance of a given network allows building a worst case scenario for examining graph vulnerability $[11,25]$. Here, we selected the most frequently used centrality measures: degree and betweenness.

Degree centrality $\left(C_{i}^{D}\right)$ measures the number of links of a node $i$, and it is known to be the simplest centrality metric for defining node importance. The idea is that important nodes have the largest number of links to other nodes [34]. In a dual mapped road network, the degree centrality is the number of neighboring roads intersecting road $i$, and is defined as [2]:

$$
C_{i}^{D}=\frac{k_{i}}{N-1}=\frac{\sum_{j \in N} a_{i j}}{N-1},
$$

where $k_{i}$ is the number of links connected to node $i$ and it is normalized by its maximum possible value of $N-1$. Using this metric, hubs are identified as nodes with extremely high $C_{i}{ }^{D}$.

Betweenness centrality $\left(C_{i}^{B}\right)$ measures the importance of a node $i$ based on how often the node lies on the shortest paths between pairs of nodes within the network. In a graph, $G$, the shortest paths between nodes $i$ and $j$ are the paths with the minimum number of links among all possible paths between them. In many cases, the shortest paths between two nodes are assumed to be the most efficient (and thus most travelled) paths between these points in a network. Although not all the flows from the origin to destination take the shortest paths in reality, betweenness has been well accepted as a good approximate metric for the influence of nodes over flow with a network [2]. Often when the nodes with high betweenness centrality are removed from the network, a significant proportion of network flow can be disrupted relative to the removal of a node with lower betweenness. The betweenness centrality is defined as $[2,35]$ :

$$
C_{i}{ }^{B}=\frac{1}{(N-1)(N-2)} \sum_{j, k \in G, j \neq k \neq i} \frac{n_{j k}(i)}{n_{j k}},
$$

where $n_{j k}$ is the number of the shortest paths between node $j$ and $k$, while $n_{j k}(i)$ is the number of shortest paths between $j$ and $k$ that passes through the node $i$.

The topological structure of a network is often characterized by using the node degree distribution $P(k)$, which is the probability of a node to have $k$ edges. The $P(k)$ of various real-world networks is often found to be a heavy-tailed distribution and are scale-free $[4,11,36]$. The node degree distribution for a scale-free network is described as:

$$
p(k) \sim k^{-\alpha}
$$

where $\alpha$ corresponds to the power-law exponent and is generally found to be $2<\alpha<3$ in real-world networks $[4,14,29]$. In general, road networks analyzed with the primal approach are not characterized by a scale-free degree distribution due to the spatial constraints of local geography that limit the expansion and growth of the connectivity of a node [1]. However, dual mapped road networks may produce power-law degree distributions due to the lack of these spatial constraints. In the analysis of dual mapped road networks, a scale-free degree distribution is often interpreted as the primary indicator for a self-organized network structure, i.e., a road network that underwent long-term growth and evolution processes without centralized control. Likewise, the lack of a scale-free degree distribution implies a road built with central, top-down planning over a short period $[14,35]$. We note that the scale-free property in node degree is not the only indicator for self-organization. For example, small-world property, clustering, street length distribution, and betweenness distribution are also measured to find the signature of self-organization of road networks [14, 35, 37]. However, those metrics have been less often used in road networks constructed by a dual approach, making those difficult to use for comparative analysis. As we aimed to detect the level of self-organization in road networks constructed by a dual approach, we thus focused on degree distribution in this study.

2.4. Vulnerability Analyses. The vulnerability of a network is assessed by evaluating the change in network properties during the removal of nodes $[11,25]$. The key topological property of interest in this work is the network efficiency which represents how efficiently material, information, or people are transported through the network [38], and is calculated as:

$$
E(G)=\frac{1}{N(N-1)} \sum_{i \neq j} \varepsilon_{i j}
$$

where $\varepsilon_{i j}$ is the inverse of the shortest path between $i$ and $j$. Crucitti et al. [39] showed that efficiency is a better measure 
of network performance than the characteristic path length because analysis results using the efficiency were more robust to the randomly chosen nodes especially when those were disconnected from the network. By measuring the degradation in the efficiency during network disruption, it is possible to evaluate how much a network can withstand relatively to a minimum or appropriate efficiency, i.e., its resilience [40].

For vulnerability analysis, networks are disrupted by successive node removal, either randomly or selectively (based on their $C_{i}^{D}$ or $C_{i}^{B}$ ), and the degradation of the efficiency is observed iteratively. The random failure mode may represent a loss in infrastructure caused by, for instances, traffic accidents, or road subsidence. Selective failure mechanisms are used in network studies to consider intentional attacks (e.g., terrorist attacks) to observe the functional response of the network under worst case scenarios. While the selective removal procedure only needs one realization as the centrality is deterministically computed, the random removal procedure requires Monte-Carlo simulation to obtain the general behavior of the network performance. Thus, we made 100 realizations for each case of random removal.

\section{Results}

3.1. Self-Organization of Road Networks. Several basic network properties, including the number of nodes $\left(N_{D}\right)$ and links $\left(M_{D}\right)$, node degree statistics (average $\langle k\rangle$, maximum degree $k_{\max }$, variance $k_{\text {var }}$, and coefficient of variation $\mathrm{CV}$ ), diameter $(D)$, characteristic path length $(L)$, and initial efficiency $\left(E_{\text {ini }}\right)$, all of which are measured in dual mapped graphs are summarized in Table 2. If one observes a network which self-organizes as it grows over time, one may possibly expect monotonic trends of above variables. For example, it is easy to imagine that $N_{D}$ and $M_{D}$ will increase over time for the growing cities [5]. Also, Kang et al. [18], by analyzing the road network of Shanghai Pudong New District from 1995 to 2007, reported increasing trends not only in the numbers of segments and intersections but also in characteristic path length, topological distance, and network efficiency. The increase in these topological metrics is attributed to the improvement of overall connectivity of the network by filling the initially emptied space with local collector roads.

However, none of the above variables clearly revealed evolution pattern of urban road networks in our case when we observed the variables of each district sorted by development history. One explanation is because the year of incorporation of a district into Seoul city does not necessarily coincide with the year of initial establishment of the road network. Also, even if it is reasonable to assume that the road network growth initiates at the time of incorporation, the rate of growth or self-organization is different by districts because of various factors such as the regional development policy of local governments. Consequently, a district which is incorporated more recently into Seoul and started to develop may have more grown and self-organized over districts established earlier. Thus, we needed to find a metric
TABLE 2: Basic network properties and statistics of the 25 urban road networks measured in the dual mapping approach.

\begin{tabular}{llllllllll}
\hline Name & $N_{D}$ & $M_{D}$ & $<k>$ & $k_{\max }$ & $k_{\text {var }}$ & $k_{\mathrm{CV}}$ & $D$ & $L$ & $E_{\text {ini }}$ \\
\hline S01 & 1520 & 2583 & 3.40 & 79 & 27.33 & 1.54 & 11 & 5.19 & 0.22 \\
S02 & 2651 & 3940 & 2.97 & 83 & 19.13 & 1.47 & 17 & 5.73 & 0.17 \\
S03 & 2525 & 3626 & 2.87 & 86 & 22.43 & 1.65 & 16 & 5.81 & 0.18 \\
S04 & 2768 & 4526 & 3.27 & 97 & 27.36 & 1.60 & 14 & 4.98 & 0.19 \\
S05 & 4630 & 7191 & 3.11 & 150 & 28.43 & 1.72 & 21 & 5.29 & 0.16 \\
S06 & 2258 & 3558 & 3.15 & 79 & 25.49 & 1.60 & 13 & 5.20 & 0.19 \\
S07 & 2122 & 3330 & 3.14 & 69 & 24.43 & 1.57 & 14 & 4.87 & 0.20 \\
S08 & 1229 & 2407 & 3.92 & 98 & 34.84 & 1.51 & 11 & 4.74 & 0.23 \\
S09 & 2081 & 4093 & 3.93 & 133 & 40.82 & 1.62 & 9 & 4.27 & 0.23 \\
S10 & 1917 & 2951 & 3.08 & 59 & 22.26 & 1.53 & 14 & 5.19 & 0.19 \\
S11 & 3491 & 5524 & 3.16 & 134 & 39.63 & 1.99 & 14 & 4.81 & 0.21 \\
S12 & 2675 & 4370 & 3.27 & 101 & 40.13 & 1.94 & 11 & 6.04 & 0.22 \\
S13 & 3575 & 5685 & 3.18 & 125 & 32.81 & 1.80 & 12 & 6.12 & 0.21 \\
S14 & 1908 & 3316 & 3.48 & 93 & 32.43 & 1.64 & 11 & 5.08 & 0.22 \\
S15 & 1559 & 2476 & 3.18 & 99 & 30.53 & 1.74 & 13 & 5.23 & 0.22 \\
S16 & 2377 & 3774 & 3.18 & 80 & 24.23 & 1.55 & 13 & 4.78 & 0.18 \\
S17 & 3194 & 5220 & 3.27 & 141 & 38.21 & 1.89 & 12 & 5.35 & 0.20 \\
S18 & 1857 & 2949 & 3.18 & 67 & 31.80 & 1.78 & 12 & 4.73 & 0.21 \\
S19 & 1170 & 2435 & 4.16 & 70 & 34.59 & 1.41 & 10 & 5.73 & 0.28 \\
S20 & 1556 & 2895 & 3.72 & 74 & 31.71 & 1.51 & 11 & 7.15 & 0.22 \\
S21 & 1690 & 3579 & 4.24 & 80 & 33.81 & 1.37 & 11 & 5.56 & 0.22 \\
S22 & 2136 & 3932 & 3.68 & 83 & 31.75 & 1.53 & 11 & 4.89 & 0.21 \\
S23 & 1800 & 3130 & 3.48 & 79 & 27.43 & 1.51 & 13 & 4.86 & 0.21 \\
S24 & 1473 & 3648 & 4.95 & 89 & 50.73 & 1.44 & 9 & 6.36 & 0.25 \\
S25 & 1806 & 3442 & 3.81 & 149 & 43.42 & 1.73 & 11 & 4.67 & 0.23 \\
\hline
\end{tabular}

which may serve as an indicator for self-organization of road networks.

As a network self-organizes, especially when this network evolves toward a scale-free network, $\langle k>$ converges to a lower value [5]. This is due to, in a dual graph, a lower growth rate of edges than that of nodes. It is a typical growth pattern in a modern road network, which includes high portion of grid-like, clustered structure with $\langle k\rangle \sim 4$ at the initial planned stage and evolves to include growing portion of tree-like, sparse structure by adding nodes (e.g., street segment) with $k=1$ or 2 at every attachment resulting in a lowered $\langle k\rangle$ [22]. This phenomenon is also observed in our datasets. The distribution of $\langle k>$ showed an increasing trend as the road history class (I to IV) increases although the trend is not clear for various reasons explained in the previous paragraph [Figure 2(a)]. We also generated a new map as in Figure 2(b), showing the gradient of self-organization of road networks categorized by $\langle k\rangle$ values. According to this map, S02 and S03 (both in Class I and $<k>s$ are smaller than 3.0) are identified as the most self-organized districts, but S01, which is also in Class I, is now classified as a less but still highly self-organized district. Indeed, majority of the districts (16 out of 25) are classified as highly selforganized with $\langle k\rangle$ larger than 3.0 and smaller than 3.5. Moreover, most of the least self-organized districts ( 8 out of 11 districts in Class IV) are now re-classified as being more self-organized than observed based on development history. This result implies that categorizing the road networks by development history does not appropriately reflect their level of self-organization. 

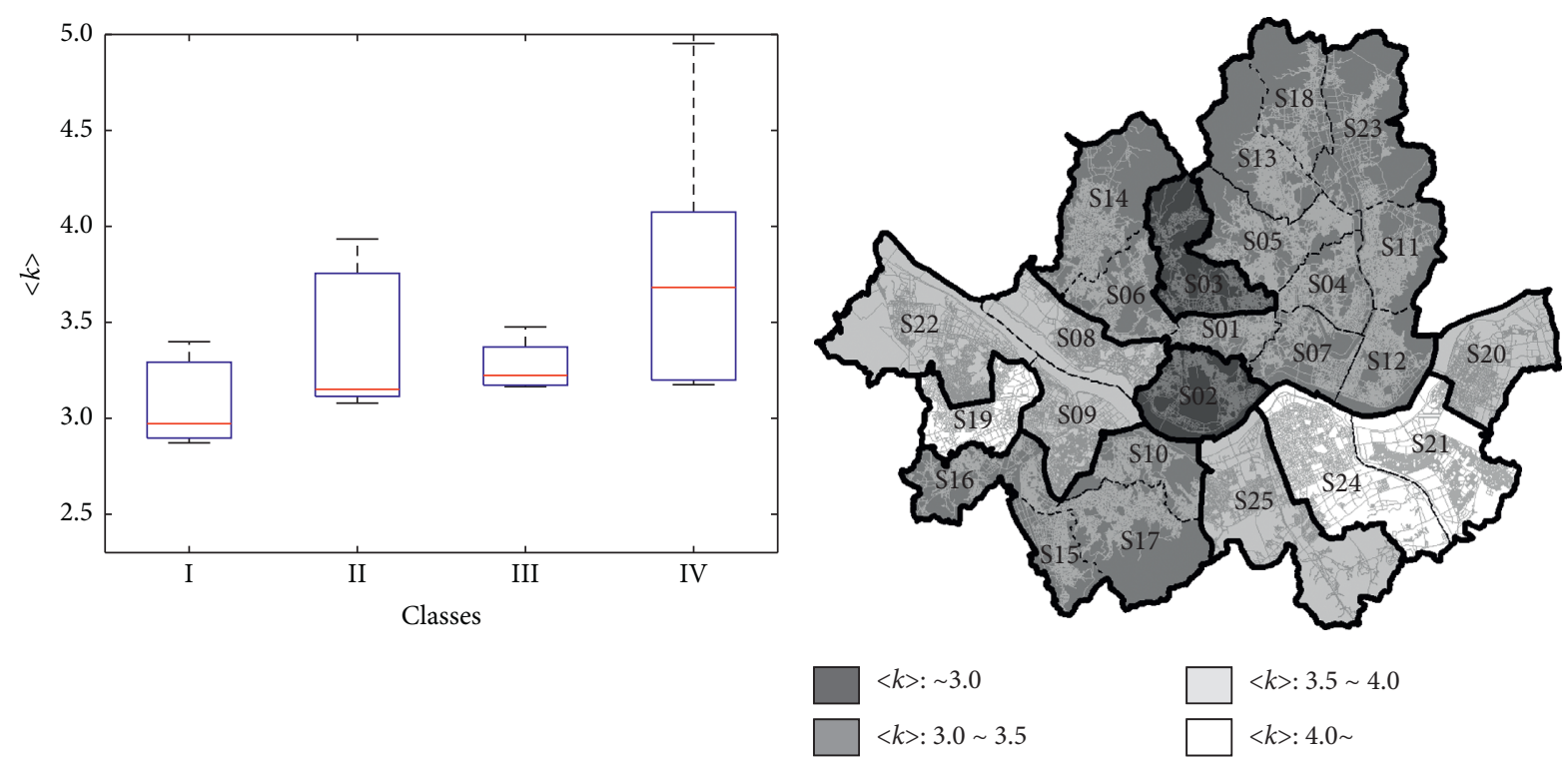

(a)

(b)

FIgURE 2: (a) Statistics of $<k>$ values for each road history class and (b) twenty-five districts in Seoul re-classified by the $<k>$ values.

From now on, we use $\langle k\rangle$ as a simple indicator for approximating relatively the level of self-organization. Given the $\langle k\rangle$ as an indicator, now we can see how the other variables in Table 2 evolve as a road network self-organizes. First, we observe that the network diameter, $D$, increases since the self-organization is accomplished by the physical growth of the network such as an addition of nodes and edges in the existing network [Figure 3(a)]. This trend is also viewed in the $E_{\text {ini }}$ which decreases as $\langle k\rangle$ decreases [Figure 3(b)]. As an efficiency is calculated by averaging the inverse of shortest path lengths of a network, a larger network would have smaller efficiency. Decreasing variance in node degrees $\left(k_{\mathrm{var}}\right)$ is another interesting observation [Figure 3(c)]. If a network grows from approximately a regular graph, which is a scale-specific, to a scale-invariant one, one would expect the $k_{\text {var }}$ to increase and sometimes to one which is unable to calculate. However, all the dual mapped road networks in this analysis already have the scale-invariance feature (this will be more explained in the next section), and are approaching a saturation phase for their physical growth due to spatial constraints. Then the growth and probability for the occurrence of hubs diminish relative to other parts of the network where space is still available for adding network compartments. Thus, decreasing probability in hub nodes combined with increasing probability in non-hub nodes results in the overall decline in the $k_{\text {var }}$. The overall shape of $k_{\max }$ values which shows no particular pattern over $\langle k\rangle$ also confirms this explanation [Figure 3(d)].

3.2. Topological Analyses. Figure 4 presents the cumulative degree distributions (CDDs) for the four road networks (S03, S07, S25, and S19) selected for representing different $\langle k\rangle$, and we report power-law exponents of the entire networks in Figure 5. We plotted the CDD because it does not require setting the binning size which sometimes produces erroneous results for estimating the slope of the fitting curves of probability distribution functions [2]. Particularly in a logarithmic scale, a logarithmic binning reduces a high noise which occurs in the tail of the distribution $[2,36]$ but it also dramatically reduces the number of data points which artificially increases the likelihood of the regression curve. Thus, we used the CDD for finding the power-law exponent of which, theoretically, the value is obtained as one smaller than the exponent obtained in non-cumulative probability distribution [2]. Also, as our purpose was to fit the powerlaw model to the entire range of the observed values instead of finding a slope on a partial range, we constrained the intercept on $y$-axis of the model to be unity (or zero on log transformed axis).

The CDDs of all road networks analyzed are well described with power-law functions, which indicate these are all scale-free networks [41]. Also, their exponent values exist in the range of approximately $1.25 \sim 1.55$ which is theoretically translated into $2.25 \sim 2.55$ when measured from noncumulative degree distributions. Note that the exponents of degree distributions of road networks are generally within the range of 2 3 and, for example, Kalapala et al. [4] reported the exponents from 2.2 to 2.4 for the dual mapped national road networks of United States, England, and Denmark. We also found an interesting pattern in their power-law exponents which increase with network selforganization measured by the decrease in $\langle k\rangle$ as presented in Figure 5. The increasing exponent demonstrates the impact of spatial constraints on the dual mapped networks. Discussion on why the exponent increases is made in Section 4.

3.3. Vulnerability Analysis. In Figure 6, node fractions removed by the random and selective procedures (both degree 


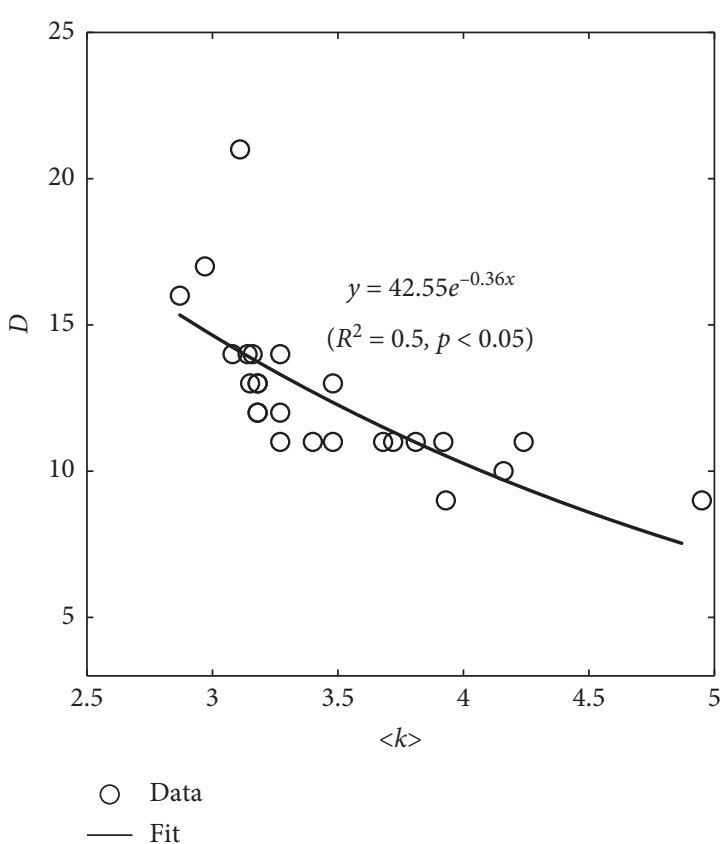

(a)

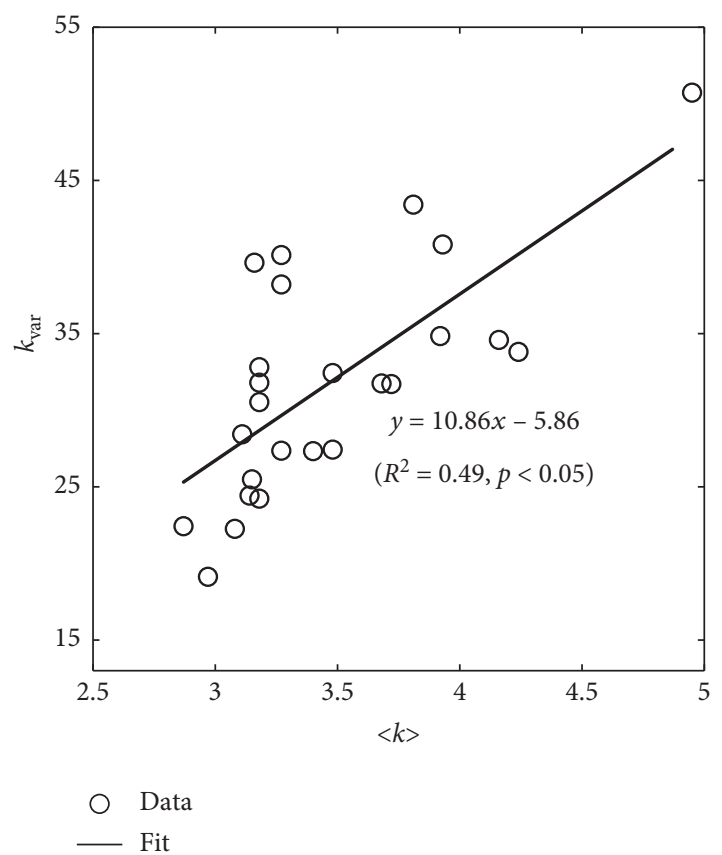

(c)

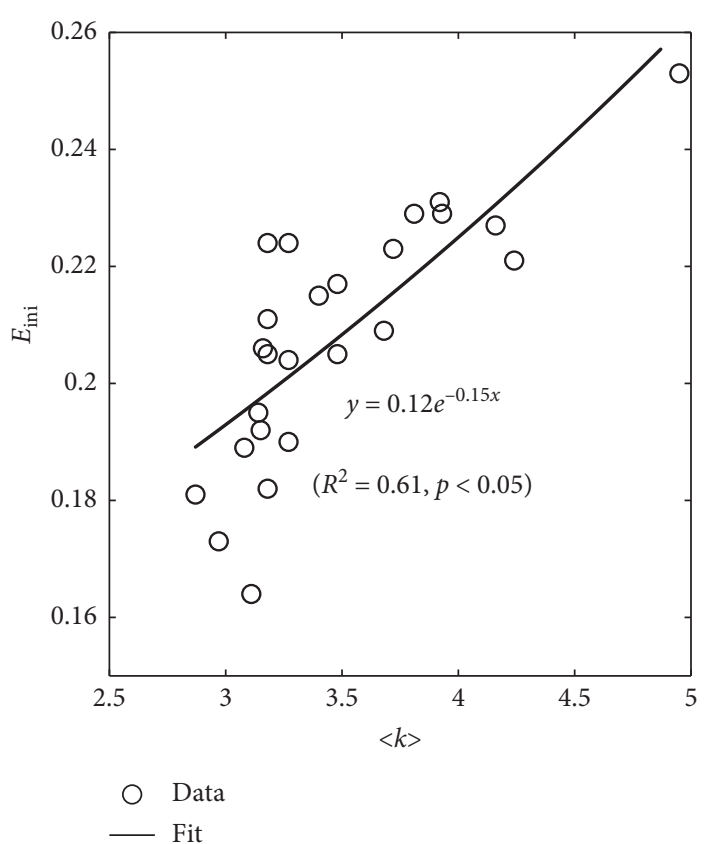

(b)

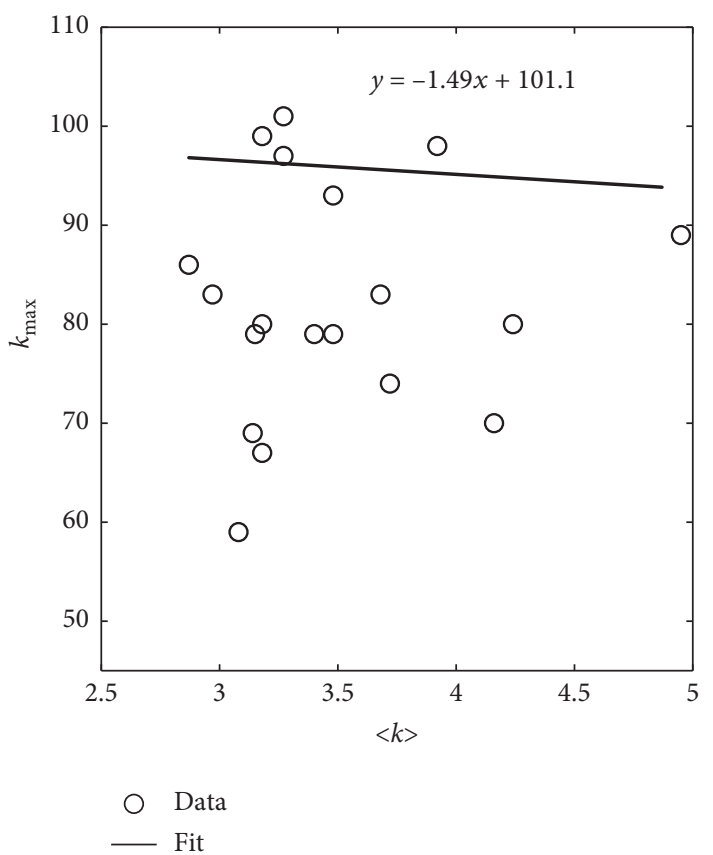

(d)

FIGURE 3: Trends of network statistics over $<k>$. (a) Diameter, (b) initial efficiency, (c) variance of $k$, and (d) maximum $k$.

and betweenness-based) until the global efficiency reached $50 \%$ of $E_{\text {ini }}$ for all road networks are plotted with respect to the $\langle k\rangle$. As the size of a network, thereby its level of selforganization, affects $E_{\text {ini }}$, we assessed the effect of node removal by calculating the reduced efficiency normalized by its initial value. The results from both random and selective removal strategies reveal that more self-organized networks indicated by decreasing $\langle k\rangle$ are more prone to both errors and attacks such that self-organized systems are more vulnerable in this analysis. However, similar to the behavior of scale-free networks [11], a larger fraction of the node [about 15 23\%, see Figure 6(a)] are required to be removed for random removal procedure while only a minor portion of nodes [less than $1 \%$ for both degree and betweennessbased attacks, see Figures 6(b) and 6(c)] are enough to reduce the efficiency significantly. Thus, even though roads are spatially embedded networks, which barely possess scalefree properties in its primal form [15], they behave similarly to scale-free networks when these are viewed in dual maps, which is also confirmed by the observation of topology. 


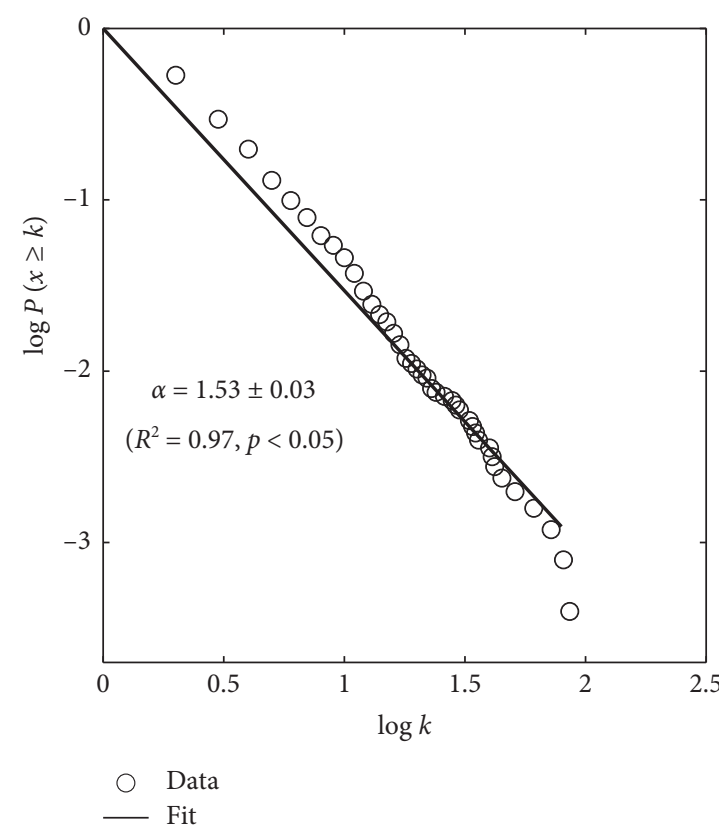

(a)

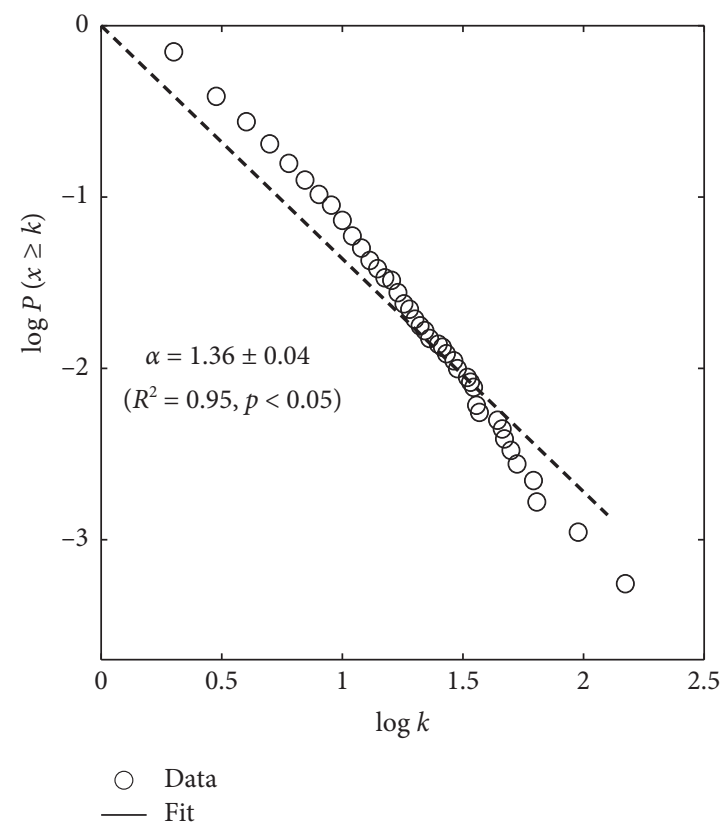

(c)

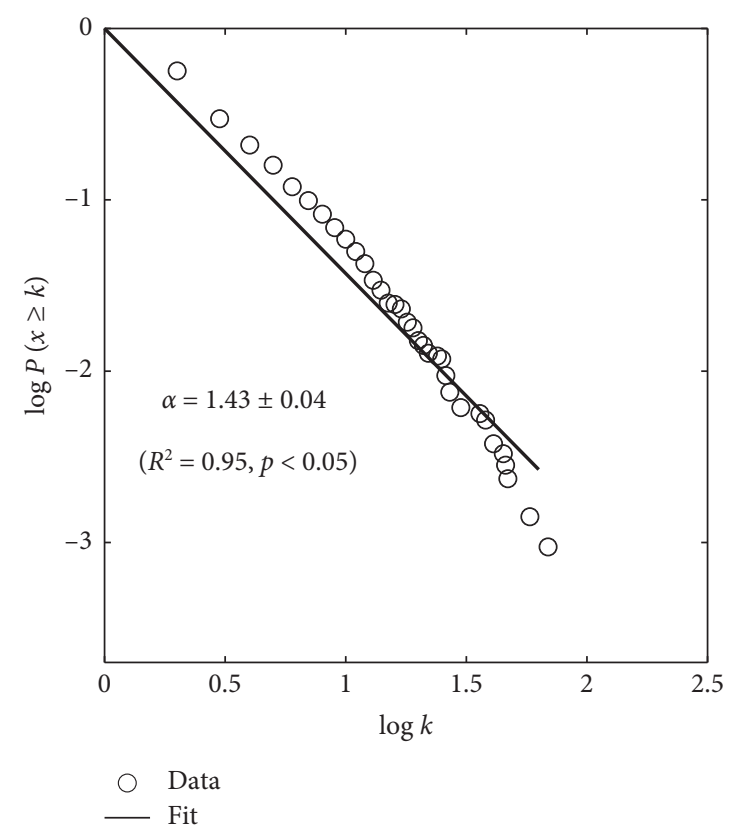

(b)

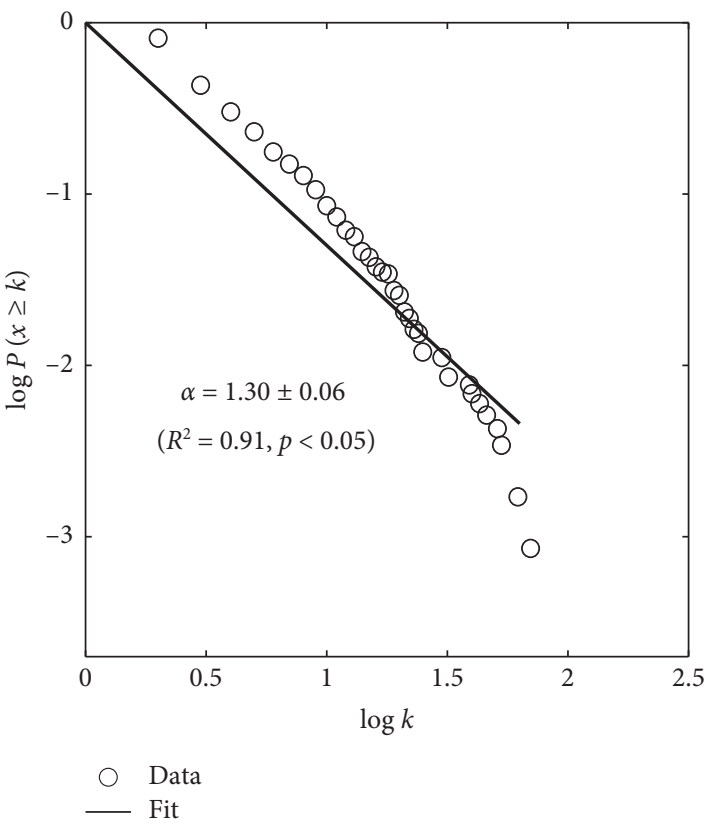

(d)

Figure 4: Cumulative degree distributions of the selected urban road networks: (a) S03, (b) S07, (c) S25, and (d) S19.

\section{Discussions}

4.1. Network Metrics. Masucci et al. [5] studied the growth and evolution of London street network over the past 224 years by analyzing the nine sets of the dual mapped network. They showed that the growth of the network, measured by the numbers of nodes and edges, was represented strikingly well by a logistic law, which implied that the London street network grew with competition for a limited space. Same observation held when this street network was analyzed with the primal graph [42]. Thus, the growth in the number of nodes and edges following a logistic law may be well observed for a growing region or city, regardless of mapping processes chosen, when it is observed over time. They also showed that the average degree decreases as a function of the number of nodes which monotonically increases over time. The difference of the average degree between the initial street network ( 5.2) and the most recent one ( 3.8) was more significant with the dual map than when it was analyzed with the primal map which only decreased from $\sim 3.3$ to 2.9. This implies that the growth of a street network, thus the level of self-organization, can be 


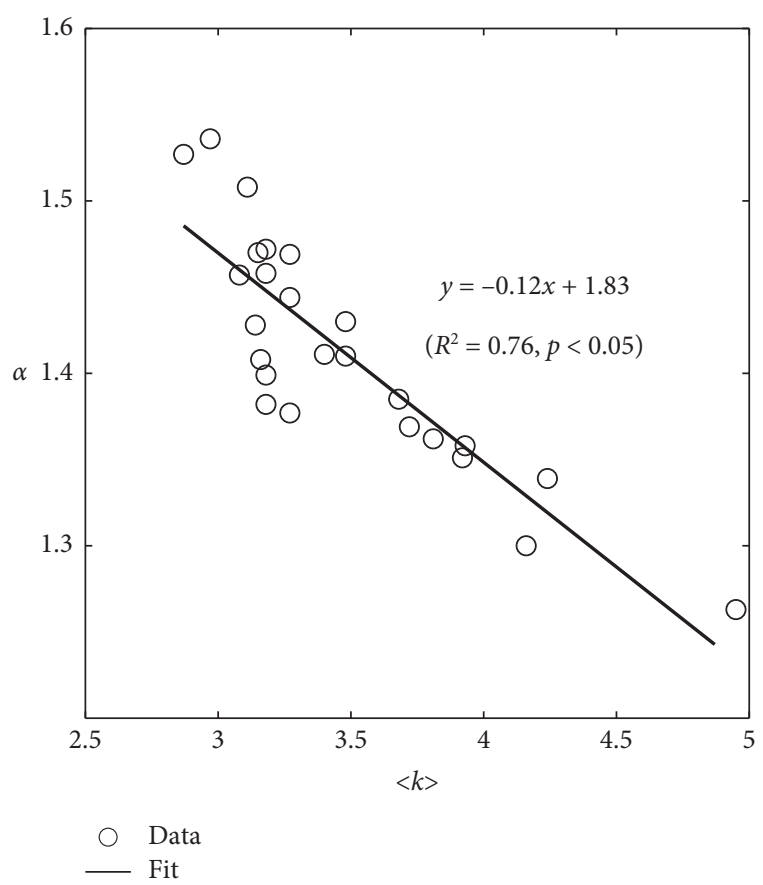

FIGURE 5: Exponents of cumulative degree distributions versus $\langle k>$ of 25 urban road networks.

captured by observing the average degree $\langle k\rangle$ especially when it is analyzed with a dual map. For examining the growth and evolution of the street network of one region, observing the number of nodes (or edges) will be enough. However, we examined the level of self-organization of multiple districts by analyzing the snapshot of the most current street map. In this case, the number of nodes (or edges) does not necessarily imply the growth level of a region due to the different areal sizes among regions, different years of regional establishment, and different driving factors that determine growth rates. Therefore, we used $\langle k\rangle$ as an alternate metric for comparing the level of growth and selforganization of multiple districts.

Using $<k>$ as an indicator, we evaluated multiple metrics including network diameter $(D)$, network efficiency $\left(E_{\text {ini }}\right)$, variance of node degree $\left(k_{\mathrm{var}}\right)$, and maximum degree $\left(k_{\max }\right)$. It is not surprising to observe the increasing trend of $D$ because a street network grows by the space filling process of minor roads, which increases the number of nodes and edges, until the limited space (e.g., within the administrative boundary of a district) is fully occupied. An interesting observation will be whether $D$ increases proportional to $\ln N$ or not, as logarithmic scaling indicates a street network as having a small-world property [43]. Although, we were not able to analyze this feature as we compared multiple districts with variable number of nodes in a snapshot, Masucci et al. [5] showed that the small-world properties of London street network was a stable attribute over the past two centuries by finding $D$ was proportional to $\ln N$.

The most interesting finding in our analysis is the decreasing trend in the variance of $k$. As was shown in Figure 4, all the street networks in the 25 districts exhibit scale-free properties. The $m$ th moment, $\left\langle k^{m}\right\rangle$, of a scale-free network diverges if $m \geq \alpha-1$ and is approximated as $\left\langle k^{m}\right\rangle \sim N^{m-\alpha+1}$ as $N \longrightarrow \infty[2]$. Thus, the second moment goes as $\left\langle k^{2}\right\rangle \sim N^{3-\alpha}$ which implies that the variance of degree keeps increasing and eventually diverges in a scale-free network. And one may also expect to observe this divergence in the street networks in Seoul since all the power-law exponents were less than three (see Figure 5). However, a street network is spatially constrained even in a dual-mapped format. Even if a spatial network loses geographical features in the dual map, its growth (e.g., adding nodes and edges) is constrained inevitably by their inherent properties as spatial networks making hub nodes to grow not beyond their geometrical space on which new neighboring nodes can attach. Thus, the entire network can grow only until the capacitated limit which is set by geographical or administrative boundary [5]. Consequently, there is a limit on how many newly developed roads can physically attach to hub roads, such that a greater number of low degree nodes can enter the system relative to the increasing degree of the hub nodes. This mechanism of fast growing frequency (or probability) in low $k$ compared to the eventual saturation of frequency in hubs (high $k$ ) also explains why $<k>$ decreases as a road network, when viewed in a dual mapping approach, grows and self-organizes.

4.2. Vulnerability of Road Networks. The existence of hubs and highly heterogeneous node degree distribution is often associated with high vulnerability to selective node removal procedures [11], and the results presented here match well to existing literature. Duan and $\mathrm{Lu}$ [44] also reported similar topological properties of six road networks in different geographic areas (in Europe, North America, and Asia), however, they found no statistically significant differences on both topology and functionality (e.g., vulnerability to errors and attacks) among these regions. On the contrary, our analysis indicates that there is a clear difference among the regions on both topology and functionality, and these differences occur as an evolution pattern of a gradual shift in the variables.

To explicitly quantify the effect of disruptions, we made a new methodology inspired by the Lorenz curve. The original Lorenz curve is a plot of the cumulative percent of a dependent variable versus the cumulative percent of an independent variable ranked from lowest to highest [45]. This plot calculates Gini coefficient for evaluating the inequality in the distribution of responses of various systems such as wealth distribution in economy [46], size distribution of individuals in ecology [47], and catchment discharge and load in hydrology [48] by comparing with a perfect equality curve (plotted as 1:1 line). As our analysis measures the response (e.g., normalized efficiency) of a network to the fraction of nodes removed from highest to a lowest degree or betweenness for the selective removal procedure, this results allow us to evaluate the inequality in a system's response. The difference is that, instead of $1: 1$ curve, we use the results from random removal (average response from 100 realizations) as the reference curve because a perfect equality in the response is highly unlikely in networked systems due to a dynamical change in the network topology during a 


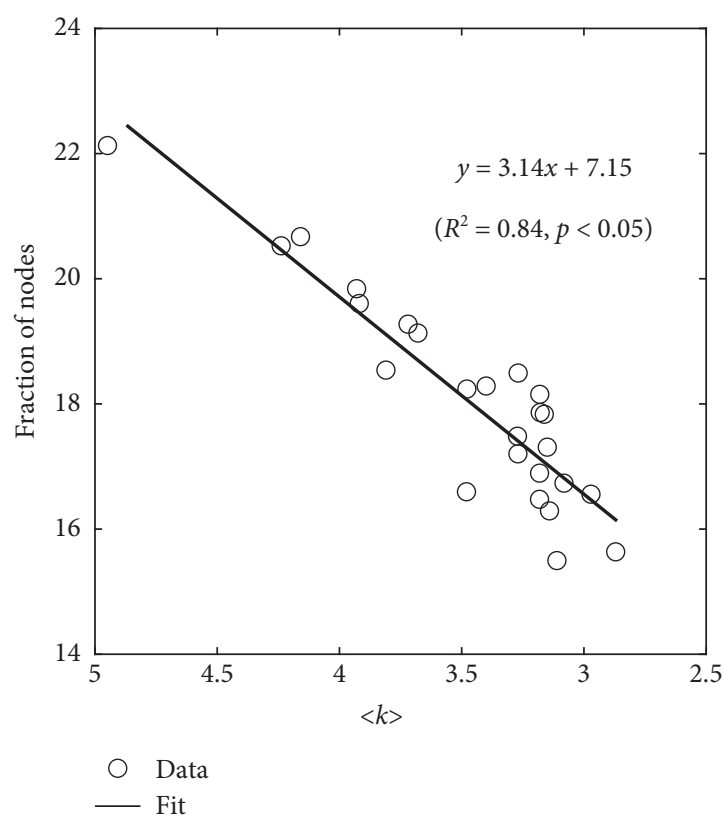

(a)

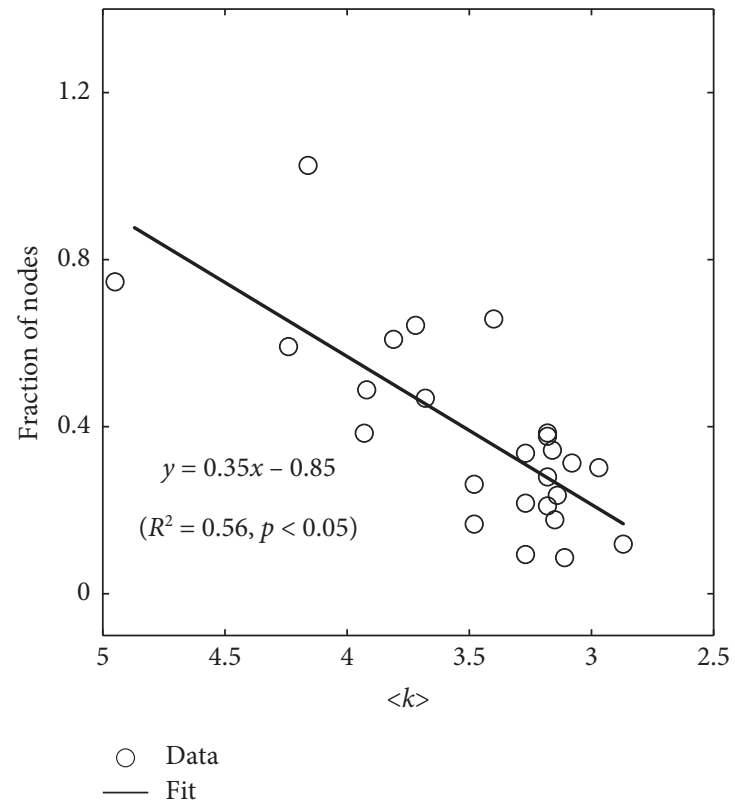

(b)

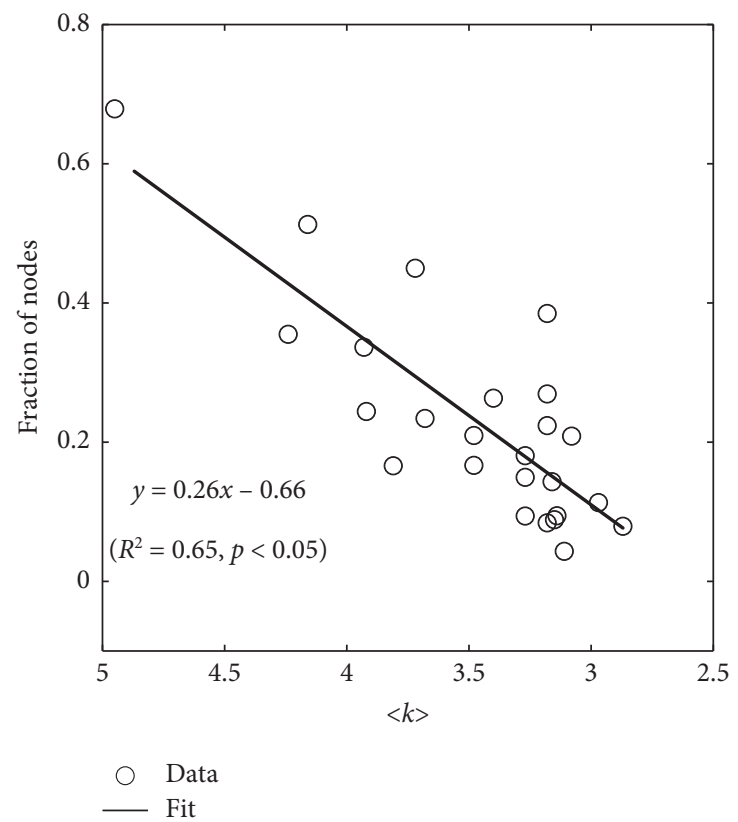

(c)

FIGURE 6: Fraction of nodes required to reduce $50 \%$ of initial efficiency over $<k>$ by (a) random removal, (b) degree-based attack, and (c) betweenness-based attack.

sequential node removal. In Figure 7, the Gini coefficient of random removal is calculated as $G_{r}=(A+B) /(A+B+C)$, and that of selective removal is $G_{s}=A /(A+B+C)$. Then, the modified Gini coefficient is expressed as $G=\left(G_{r}-G_{s}\right) / G_{r}=B /(A+B)$.

Figure $8(\mathrm{a})$ and 8 (b) present $G$ values of all road networks measured on the degree $\left(G_{D}\right)$ and betweenness $\left(G_{B}\right)$ based attacks, respectively. The range of $G$ is narrow $\left(G_{D}: 0.93 \sim 0.99\right.$ and $\left.G_{B}: 0.95 \sim 0.99\right)$, but it is clear that both $G$ values increase with decreasing $\langle k\rangle$. The small ranges in $G$ indicate that all road networks already have significantly grown for several decades, and these are all characterized by self-organized networks which are also presented in Figures 4 and 5. Although a smaller $\langle k\rangle$ already implies the corresponding network to be more self-organized, a greater $G$ also tells that the attack tolerance is lower which is a typical response of a scale-free network especially when compared to an ER random network (see Albert et al. [11]; for example).

To support our argument, we also additionally analyzed a road network in Sejong city which began its establishment 


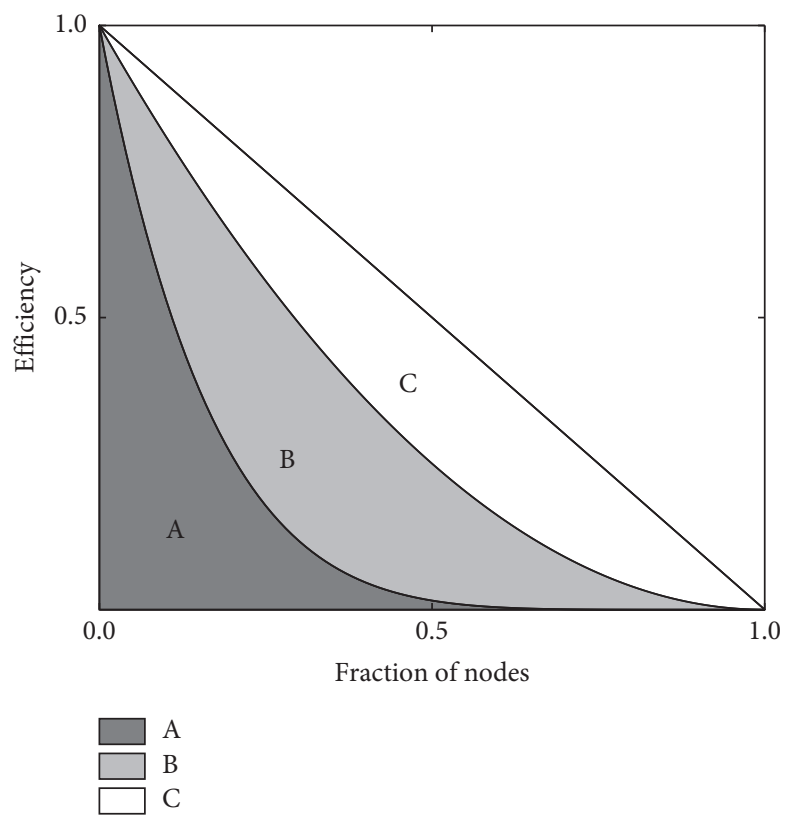

FIgURE 7: The diagram for calculating the modified Gini coefficient.

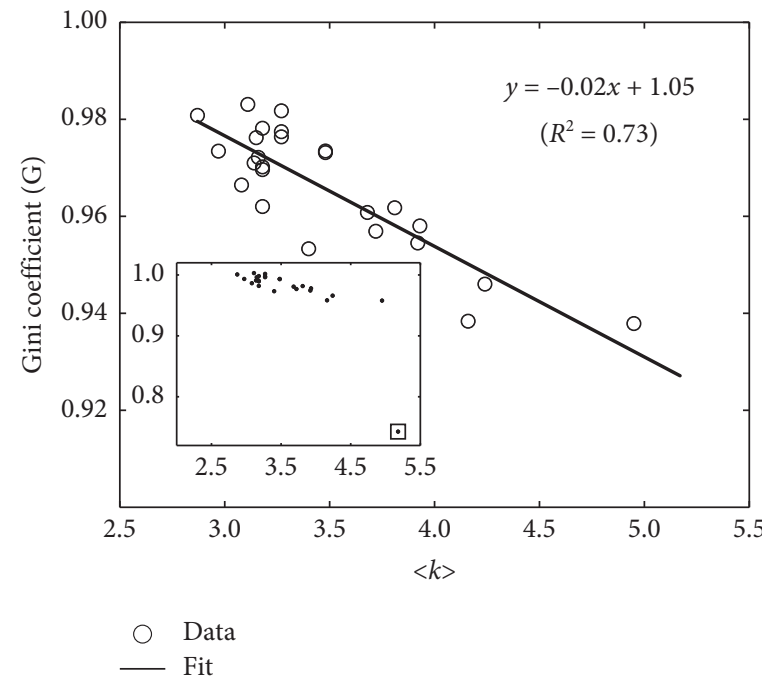

(a)

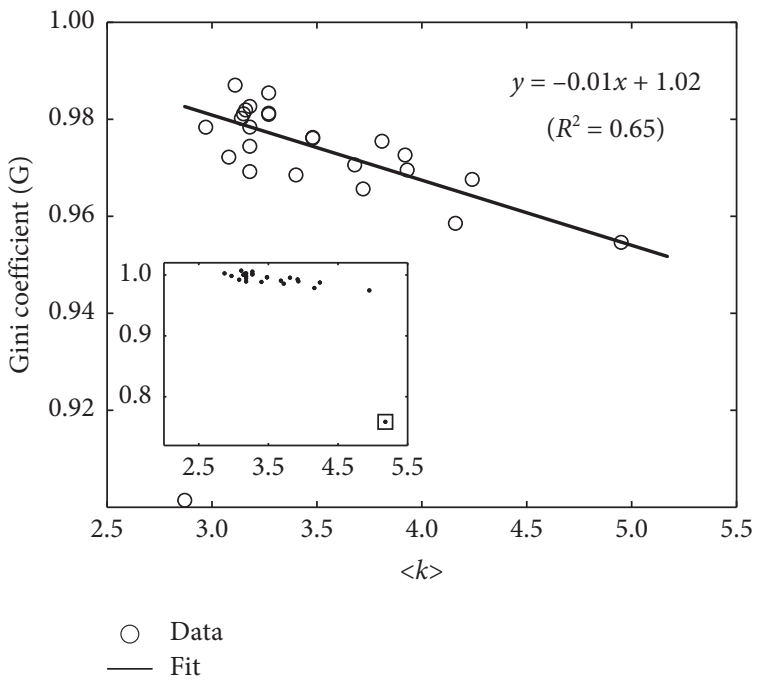

(b)

Figure 8: Modified Gini coefficients versus $<k>$ measured by (a) degree-based attack $\left(G_{D}\right)$ and (b) betweenness-based attack $\left(G_{B}\right)$.

in 2012 to distribute population and central administrative agencies outside of Seoul. Since it has grown only for a few years, we hypothesized that the road network of Sejong city would show its characteristics far from the road networks in Seoul. As in Figure 9, the $p d f$ of $k$ in Sejong city has a shorter tail with $k_{\max }=21$ and is better described with an exponential distribution function which also indicates a limited probability in low $k$ values. On the other hand, $k_{\max }$ value of 25 districts in Seoul ranged from 59 to 150 (see Table 2). Consequently, the $<k>$ and $G_{D}$ for Sejong city are 5.18 (the greatest among all) and 0.72 (the smallest among all), respectively (see insets of Figure 8). These results confirm our hypothesis that a growing road network begins with high $<k>$ and gradually shift to a low value as it evolves. Also, due to its less self-organized pattern, thus being closer to an ER random network than to a scale-free network, the effect of selective disruptions compare to random disruptions measured as $G$ is smaller.

\section{Conclusions}

As some urban systems such as Seoul transition from centralized, top-down planning to spatially distributed, decentralized growth and evolution, the resulting infrastructure networks will have shifting topologies that must be measured to understand how failures influence urban 


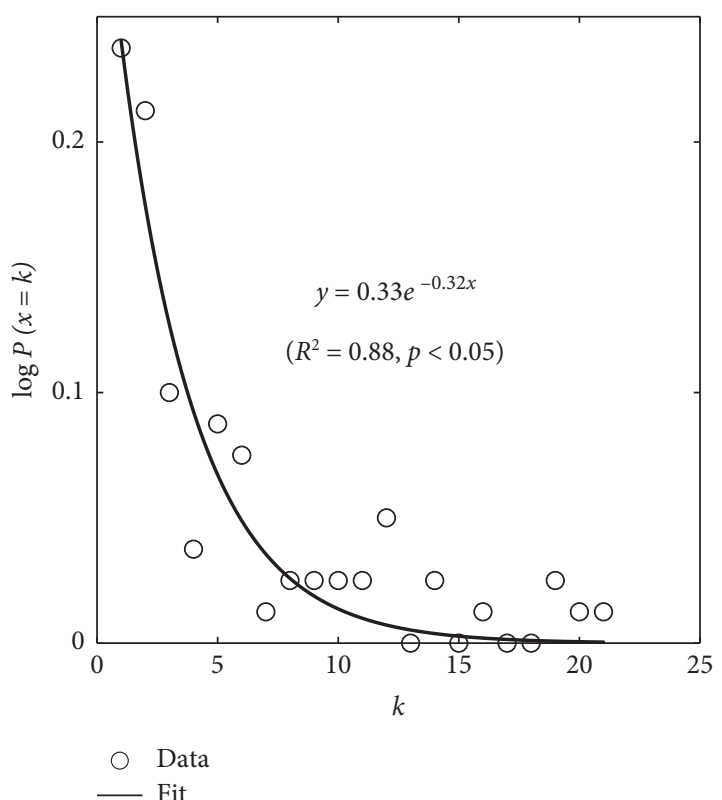

Figure 9: Degree distribution of the road network in Sejong city fitted with an exponential distribution function.

resilience and sustainability. In this work, we studied 25 urban road networks in Seoul to identify which distinct topological features are relevant for measuring network growth and vulnerability. The dual mapping method was successful in revealing distinct topological features, and our results showed that all road networks, which have undergone their growth for decades, exhibit power-law degree distributions and scale-invariance in node degree. Moreover, roads characterized by power-law degree distribution show an increasing trend in their exponents as the network grows and evolves. We propose that this ordering arises from the spatial constraints in which road networks grow with hub roads are inevitably limited by physical space and cost compared to the introduction of new roads to non-hubs with available space for filling.

We then analyzed the vulnerability of the networks to static failure scenarios to relate road network structure to function. In this analysis, the more self-organized road networks were found to be more vulnerable to both random and selective node removal methods. This contradicts the general notion that self-organized, complex systems are vulnerable to selective failures and more resilient to random failures [49]. Moreover, we proposed a new method, inspired by Lorenz curve, for quantifying network vulnerability regarding the level of self-organization. This modified Lorenz curve enables calculating the relative impact of intensive attacks to random failures and shows that a more self-organized road network tends to become more vulnerable to selective attacks.

Overall, applying complex network theory to Korean road networks has revealed numerous topological and dynamic features of the infrastructure otherwise overlooked. In particular, the methods and analyses in this work can be used as a starting point to estimate the impacts of failures on civil infrastructure systems. Moreover, the results from network analyses provides discourse on the relationship between increasing population and densification of urban areas and the increasing complexity of infrastructure systems that comprise them. In addition, this study applied complex network theory to empirical Korean road data, which has not been done in literature to our knowledge. Finally, while numerous studies on road networks investigated the universality of network topological structure, few studied the vulnerability of road networks. Taken together, this work provides a scientific way of finding critical roads in an urban system, and characterizing their vulnerabilities to different types of failures. This information is particularly useful for testing the tolerance of existing roads to disaster scenarios or inform the design of future infrastructure to prepare for and reduce the consequences of these disasters.

In addition to the current analysis, there are several ways in which to improve results in future work. First, future work should use real flow data to identify critical roads and develop more realistic methods for evaluating network vulnerability. Here, we analyzed the vulnerability based on network betweenness centrality. Although, betweenness is a well-recognized approximation of transportation flow in a network, it may not necessarily coincide with real flows because of local heterogeneity such as speed limits and land use. Second, a different methodology for node removal may be required to better reflect road failures. Since the dual mapping approach defines an assemblage of continuous road segments as a single node, node removal can imply a larger-than-realistic disruption to the network that forces numerous intersections to respond homogeneously. In reality, however, random disruptions such as local flooding or road subsidence only affect transportation locally within a road segment or intersection. Using a methodology which acknowledges such failure conditions may improve model realism.

\section{Data Availability}

The data used to support the findings of this study are available from the corresponding author upon request.

\section{Conflicts of Interest}

The authors declare that they have no conflicts of interest.

\section{Acknowledgments}

This research was supported by Basic Science Research Program through the National Research Foundation of Korea (NRF) funded by the Ministry of Science and ICT (NRF-2019R1C1C1008017 and NRF-2016R1C1B1011770). The authors acknowledge the contribution of Daniel Eisenberg (ASU) who provided substantive and helpful review.

\section{References}

[1] M. Barthélemy, "Spatial networks," Physics Reports, vol. 499, no. 1-3, pp. 1-101, 2011. 
[2] M. Newman, Networks: An Introduction, Oxford University Press, Oxford, UK, 2010.

[3] J. Buhl, J. Gautrais, N. Reeves et al., "Topological patterns in street networks of self-organized urban settlements," The European Physical Journal B, vol. 49, no. 4, pp. 513-522, 2006.

[4] V. Kalapala, V. Sanwalani, A. Clauset, and C. Moore, "Scale invariance in road networks," Physical Review E, vol. 73, no. 2, Article ID 026130, 2006.

[5] A. P. Masucci, K. Stanilov, and M. Batty, "Exploring the evolution of London's street network in the information space: a dual approach," Physical Review E, vol. 89, no. 1, Article ID 012805, 2014.

[6] S. Wang, D. Yu, M.-P. Kwan, H. Zhou, Y. Li, and H. Miao, "The evolution and growth patterns of the road network in a medium-sized developing city: a historical investigation of changchun, China, from 1912 to 2017," Sustainability, vol. 11, no. 19 , p. $5307,2019$.

[7] R. Albert, I. Albert, and G. L. Nakarado, "Structural vulnerability of the North American power grid," Physical Review E, vol. 69, no. 2, Article ID 025103, 2004.

[8] D. A. Eisenberg, J. Park, and T. P. Seager, "sociotechnical network analysis for power grid resilience in South Korea," Complexity, vol. 2017, Article ID 3597010, 14 pages, 2017.

[9] D. H. Kim, D. A. Eisenberg, Y. H. Chun, and J. Park, "Network topology and resilience analysis of South Korean power grid," Physica A: Statistical Mechanics and its Applications, vol. 465, pp. 13-24, 2017.

[10] A. Yazdani and P. Jeffrey, "Complex network analysis of water distribution systems," Chaos: An Interdisciplinary Journal of Nonlinear Science, vol. 21, no. 1, Article ID 016111, 2011.

[11] R. Albert, H. Jeong, and A.-L. Barabási, "Error and attack tolerance of complex networks," Nature, vol. 406, no. 6794, pp. 378-382, 2000.

[12] Y. Casali and H. R. Heinimann, "A topological analysis of growth in the Zurich road network," Computers, Environment and Urban Systems, vol. 75, pp. 244-253, 2019.

[13] S. H. Y. Chan, R. V. Donner, and S. Lämmer, "Urban road networks-spatial networks with universal geometric features?" The European Physical Journal B, vol. 84, no. 4, pp. 563-577, 2011.

[14] B. Jiang, "A topological pattern of urban street networks: universality and peculiarity," Physica A: Statistical Mechanics and Its Applications, vol. 384, no. 2, pp. 647-655, 2007.

[15] S. Lämmer, B. Gehlsen, and D. Helbing, "Scaling laws in the spatial structure of urban road networks," Physica A: Statistical Mechanics and Its Applications, vol. 363, no. 1, pp. 89-95, 2006.

[16] E. Strano, V. Nicosia, V. Latora, S. Porta, and M. Barthélemy, "Elementary processes governing the evolution of road networks," Scientific Reports, vol. 2, pp. 1-8, 2012.

[17] M. Barthélemy, P. Bordin, H. Berestycki, and M. Gribaudi, "Self-organization versus top-down planning in the evolution of a city," Scientific Reports, vol. 3, no. 1, p. 2153, 2013.

[18] L. Kang, C. Yang, J. C. Peters, and P. Zeng, "Empirical analysis of road networks evolution patterns in a government-oriented development area," Environment and Planning B: Planning and Design, vol. 43, no. 4, pp. 698-715, 2016.

[19] J. Wang, "Resilience of self-organised and top-down planned cities-A case study on london and Beijing street networks," PLoS One, vol. 10, no. 12, Article ID 0141736, 2015.

[20] F. Blumensaat, M. Wolfram, and P. Krebs, "Sewer model development under minimum data requirements," Environmental Earth Sciences, vol. 65, no. 5, pp. 1427-1437, 2012.
[21] M. Mair, J. Zischg, W. Rauch, and R. Sitzenfrei, "Where to find water pipes and sewers?-On the correlation of infrastructure networks in the Urban environment," Water, vol. 9, no. 2, p. 146, 2017.

[22] J. Zischg, C. Klinkhamer, X. Zhan, P. S. C. Rao, and R. Sitzenfrei, "A century of topological coevolution of complex infrastructure networks in an Alpine City," Complexity, vol. 2019, Article ID 2096749, 16 pages, 2019.

[23] Y. Kim, J. Suh, J. Cho, S. Singh, and J. Seo, "Development of real-time pipeline management system for prevention of accidents," International Journal of Control and Automation, vol. 8, no. 1, pp. 211-226, 2015.

[24] P.-J. Kwak, S.-H. Park, C.-H. Choi, H.-D. Lee, J.-M. Kang, and I.-H. Lee, "IoT (internet of things)-based underground risk assessment system surrounding water pipes in Korea," Advanced Science and Technology Letters, vol. 99, pp. 23-26, 2015.

[25] P. Crucitti, V. Latora, M. Marchiori, and A. Rapisarda, "Error and attack tolerance of complex networks," Physica A: Statistical Mechanics and Its Applications, vol. 340, no. 1-3, pp. 388-394, 2004.

[26] X. Zhan, S. V. Ukkusuri, and P. S. C. Rao, "Dynamics of functional failures and recovery in complex road networks," Physical Review E, vol. 96, no. 5, Article ID 052301, 2017.

[27] F. Xie and D. Levinson, "Topological evolution of surface transportation networks," Computers, Environment and Urban Systems, Elsevier, vol. 33, no. 3, , pp. 211-223, 2009.

[28] S. Porta, P. Crucitti, and V. Latora, "The network analysis of urban streets: a primal approach," Environment and Planning B: Planning and Design, vol. 33, no. 5, pp. 705-725, 2006.

[29] S. Porta, P. Crucitti, and V. Latora, "The network analysis of urban streets: a dual approach," Physica A: Statistical Mechanics and Its Applications, vol. 369, no. 2, pp. 853-866, 2006.

[30] M. Rosvall, A. Trusina, P. Minnhagen, and K. Sneppen, "Networks and cities: an information perspective," Physical Review Letters, vol. 94, no. 2, Article ID 028701, 2005.

[31] B. Jiang and C. Claramunt, "Topological analysis of urban street networks," Environment and Planning B: Planning and Design, vol. 31, no. 1, pp. 151-162, 2004.

[32] C. Lagesse, P. Bordin, and S. Douady, "A spatial multi-scale object to analyze road networks," Network Science, vol. 3, no. 1, pp. 156-181, 2015.

[33] B. Jiang, Axwoman 6.3: An ArcGIS Extension for Urban Morphological Analysis, University of Gävle, Sweden, University of Gävle, Sweden, Europe, 2015, http://fromto.hig.se/ $\sim$ bjg/Axwoman/.

[34] V. Latora and M. Marchiori, "A measure of centrality based on network efficiency," New Journal of Physics, vol. 9, no. 6, p. 188, 2007.

[35] P. Crucitti, V. Latora, and S. Porta, "Centrality measures in spatial networks of urban streets," Physical Review E, vol. 73, no. 3, Article ID 036125, 2006.

[36] A. Clauset, C. R. Shalizi, and M. E. J. Newman, "Power-law distributions in empirical data," SIAM Review, vol. 51, no. 4, pp. 661-703, 2009.

[37] G. Haslett, S. Bullock, and M. Brede, "Planar growth generates scale-free networks," Journal of Complex Networks, vol. 4, no. 4, pp. 500-516, 2016.

[38] V. Latora and M. Marchiori, "Efficient behavior of smallworld networks," Physical Review Letters, vol. 87, no. 19, Article ID 198701, 2001.

[39] P. Crucitti, V. Latora, M. Marchiori, and A. Rapisarda, "Efficiency of scale-free networks: error and attack tolerance," 
Physica A: Statistical Mechanics and Its Applications, vol. 320, pp. 622-642, 2003.

[40] J. Park, T. P. Seager, and P. S. C. Rao, "Understanding resilience of complex value-chain networks," in Sustainable Value Chain Management: A Research Anthology, Chapter: 19, A. Lindgreen, F. Maon, J. Vanhamme, and S. Sen, Eds., Gower Publishing, Ltd., Surrey, UK, 2013.

[41] A.-L. Barabási and R. Albert, "Emergence of scaling in random networks," Science, vol. 286, no. 5439, pp. 509-512, 1999.

[42] A. P. Masucci, K. Stanilov, and M. Batty, "Limited urban growth: london's street network dynamics since the 18th century," PLoS One, vol. 8, no. 8, 2013.

[43] D. Watts and S. Strogatz, "Collective dynamics of smallworld'networks," Nature, vol. 393, pp. 440-442, 1998.

[44] Y. Duan and F. Lu, "Robustness of city road networks at different granularities," Physica A: Statistical Mechanics and Its Applications, vol. 411, pp. 21-34, 2014.

[45] J. L. Gastwirth, "A general definition of the Lorenz curve," Econometrica, vol. 39, no. 6, pp. 1037-1039, 1971.

[46] M. O. Lorenz, "Methods of measuring the concentration of wealth," Publications of the American Statistical Association, vol. 9, no. 70, p. 209, 1905.

[47] C. Damgaard and J. Weiner, "Describing inequality in plant size or fecundity," Ecology, vol. 81, no. 4, pp. 1139-1142, 2000.

[48] H. Gall, J. Park, C. Harman, J. Jawitz, and P. S. Rao, "Landscape filtering of hydrologic and biogeochemical responses in managed catchments," Landscape Ecology, vol. 28, no. 4, pp. 651-664, 2013.

[49] A. Tero, S. Takagi, T. Saigusa et al., "Rules for biologically inspired adaptive network design," Science, vol. 327, no. 5964, pp. 439-442, 2010. 